\title{
Raumpolitik meets Aussenpolitik BEI MAKROREGIONALEN EU-STRATEGIEN \\ Praxiserfahrungen im Umgang mit einem innovativen europäischen Politikinstrument an der Schnittstelle unterschiedlicher Logiken
}

\author{
Roland ArbTER, Wien*
}

\section{INHALT}

1 Die ,produktive Unruhe“ in der supranationalen Zusammenarbeit hält seit dem Beschluss der ersten makroregionalen EU-Strategie zum Ostseeraum im Jahr 2009 weiter an

2 Die bisherigen Methoden einer Analyse und Bewertung der Wirkungen makroregionaler EU-Strategien kämpfen mit komplexen Systemschnittstellen

3 Ausblick: Wieviel Offenheit benötigen und wie viel Verbindlichkeit vertragen makroregionale EU-Strategien?

4 Schlussbetrachtung: Positive Emotionalität als Antwort auf die hohe Komplexität in der transnationalen Zusammenarbeit?

5 Literaturverzeichnis

\section{Summary}

Spatial policy meets foreign policy in macro-regional strategies of the EU

Macro-regional EU-strategies have since their launching in 2009 led to a "stimulating unrest" in the field of transnational cooperation. These pilot-type processes follow the characteristic of soft policy tools and are applying a multi-level and cross-sectoral approach at the crossroads of territorial policies and the arena of foreign relations. The author takes stock of the debate about results, impacts and value added so far from the perspective of the national coordination in Austria.

\footnotetext{
* Mag. Roland ArbTer (Dipl.-Geograph und Raumplaner ETH Zürich), Bundeskanzleramt, Abteilung Koordination für Raumordnung und Regionalpolitik, Ballhausplatz 2, A-1014 Wien; E-Mail: roland. arbter@bka.gv.at, http://www.bka.gv.at
} 


\section{Zusammenfassung}

Makroregionale EU-Strategien haben seit ihrer Einführung im Jahr 2009 zu einer „produktiven Unruhe“ im Bereich der transnationalen Zusammenarbeit in Europa geführt. Die bisherigen durchwegs noch pilothaften Ansätze verstehen sich als sanfte, mehrere Ebenen und Sektoren übergreifende Politikinstrumente an der Schnittstelle von Außen- und raumbezogener Fachpolitik. Der Artikel beleuchtet die aktuelle Diskussion zu Fragen der Wirkungen und des Mehrwerts makroregionaler EU-Strategien aus der Sicht der nationalen Koordination in Österreich.

\section{Die ,produktive Unruhe“" in der supranationalen Zusammen- arbeit hält seit dem Beschluss der ersten makroregionalen EU-Strategie zum Ostseeraum im Jahr 2009 weiter an}

Bis zur Mitte des Jahres 2014 wurden in Europa vier makroregionale Strategieprozesse lanciert (vgl. Website der EU-Kommission und Website der ÖROK). Jeweils im Auftrag des Europäischen Rates und der Regierungschefs der Europäischen Union (EU) legte die Europäische Kommission bis dato für den Ostseeraum (2009), den Donauraum (2011) und für den Adriatisch-Ionischen Raum (2014) jeweils ein Strategiedokument samt Aktionsplan vor und leitete diese an den Europäischen Rat, das Europäische Parlament, den Ausschuss der Regionen und an den Wirtschafts- und Sozialausschuss der EU als Politikempfehlung weiter. Ziel dieser Strategien ist eine im EU-Kontext verstärkte Koordinierung der teilnehmenden Staaten und Regionen zu einer breiten Palette an Kooperationsthemen. Im Juni 2015 soll die EU-Strategie für den Alpenraum als vierte makroregionale EU-Strategie folgen.

Das Konzept der makroregionalen EU-Strategien hat mehrere Väter und Mütter. Die Ursprünge finden sich sowohl in den geo-, außen- und integrationspolitischen Ansätzen staatenübergreifender Zusammenarbeit in Europa seit dem Zweiten Weltkrieg als auch in der grenzüberschreitenden und europäischen Raum- und Regionalentwicklungspolitik (DuBoIs et al. 2009). Während der Einsatz des Instrumentariums im Bereich der internationalen Beziehungen in der Regel einer außenpolitischen oder sektoralen Logik folgt (z.B. zu Themen wie Sicherheit, Handel, Energie), sprechen die diversen supranationalen Konzepte der Raum- und Regionalentwicklung transnationale Kooperationsbedürfnisse und -potenziale als integrierte Fachbereiche und Fachebenen übergreifende Herausforderungen an.

Die raumentwicklungspolitische Zusammenarbeit in staatenübergreifenden Räumen begann bereits in den 1950er Jahren in zahlreichen europäischen Grenzräumen und führte in den 1980er und 1990er Jahren zu ersten Leitbildern und Strategien für die räumliche Entwicklung europäischer Großregionen, die dann den Nährboden für das Europäische Raumentwicklungskonzept 1999 bildeten (GöRMAR 2010). Der Konnex mit den Förderprogrammen der EU-Kohäsionspolitik seit den 1990er Jahren (vor allem in Form der EU-Gemeinschaftsinitiativen INTERREG I, II und III im Zeitraum 
1990-2007 sowie der Programme der Europäischen Territorialen Zusammenarbeit [ETZ] 2007-2013) führte zu einer verstärkten Umsetzungs- und Projektorientierung in der transnationalen regionalpolitischen Kooperation bei teilweiser Abkoppelung von den traditionellen Formen der zwischenstaatlichen Zusammenarbeit.

Der Bedeutungsgewinn des EU-Ziels ,Territorialer Zusammenhalt“ (EuropäIsche Kommission 2008) stärkte zwar grundsätzlich die konzeptive Grundlage für funktionalräumliche, administrative Grenzen übergreifende Politiken und bildete auch eine Referenz für die Neugestaltung der EU-Kohäsionspolitik 2014-2020 im Sinne des forcierten ,place-based approach“, führte jedoch bis dato nicht zu einer kohärenten, Sektoren übergreifenden Politik der EU hinsichtlich des Territorialen Zusammenhalts.

Letztlich ist der Auslöser für die Einführung makroregionaler EU-Strategien stärker bei der Außen- und Integrationspolitik als im Diskurs zur EU-Raumentwicklungspolitik zu suchen, der in den letzten zehn Jahren an Bedeutung verloren hat. Zu Beginn der neuen EU-Förderperiode 2014-2020 wird verstärkt versucht, den Konnex der makroregionalen Strategien mit der EU-Kohäsionspolitik zu finden, wobei man sich hier auch auf das Konzept des Territorialen Zusammenhalts bezieht.

Makroregionale Strategien basieren auf keinem expliziten eigenen EU-Rechtsrahmen. Bisher wird ihr Mandat primär aus politischen Beschlüssen des Europäischen Rates der Regierungschefs und des Rates abgeleitet. Die Europäische Kommission agiert im Auftrag der Staaten und nimmt eine im Detail nicht definierte strategische Koordinationsfunktion wahr. Ziel ist eine verstärkte Zusammenarbeit zur Bewältigung gemeinsamer Herausforderungen in einem bestimmten geographischen Raum (S AMIECKi 2009).

Das Konzept erfordert als Ausgangsbasis quasi ein Gefühl regionaler Identität, den Wunsch nach gemeinsamer strategischer Planung und die Bereitschaft zur Bündelung von Ressourcen. Der methodische Doppelansatz eines Strategiedokuments, das Bezüge zu den zentralen EU-Zielen herstellt (z.B. EU 2020, EU-Erweiterung, etc.), und eines Aktionsplans als Umsetzungsrahmen, der für einen längerfristig ausgerichteten kontinuierlichen Kooperationsprozess messbare operative Ziele formuliert und konkrete Projektvorhaben anspricht (ohne dafür neue finanzielle Mittel bereit zu stellen), geht ausdrücklich auf bisherige Defizite sowohl in der zwischenstaatlichen Außenpolitik (wie fehlende konkrete Umsetzungsergebnisse) wie auch in der grenzübergreifenden Raum- und Regionalentwicklung ein. Solche Defizite bestehen einerseits in der Gefahr eines Strategieüberhangs bei den klassischen Raumordnungskooperationen und andererseits in jener von wenig in Strategien eingebetteten Projektförderungen in den INTERREG- und ETZ-Programmen.

Der Brückenschlag zwischen hochrangig politisch bestimmter Strategiearbeit und konkreten Umsetzungsaktionen bietet neue Möglichkeiten für Kooperationen. Die Offenheit des Ansatzes und sein Charakter eines sanften Politikinstruments bergen sowohl Chancen als auch Gefahren. Gerade die Möglichkeit der flexiblen Anpassung an den jeweiligen Kontext führte jedoch zu einer raschen und breiten Akzeptanz bei den Akteuren. Als eine nicht abschießend definierte Politik (fuzzy concept) eröffnen makroregionale Strategien Raum für unterschiedliche Einschätzungs- und Interpretationsmöglichkeiten. Neben der prominenten Rolle der EU-Kommission in 
makroregionalen Strategieprozessen und der erhöhten politischen Aufmerksamkeit bildet gerade die Möglichkeit dieser visionären Vorwegnahme von Folgewirkungen und Nutzenerwartungen wahrscheinlich eine der Motivationsquellen für Promotoren, makroregionale Strategieprozesse voranzutreiben. Hierbei reichen die Vorstellungen von europapolitischen Visionen für teilräumlich differenziertere EU-Politiken bis zu neuen Potenzialen für stärker strategisch ausgerichtete Steuerungsmodelle in der EU-Kohäsionspolitik oder zu einem präferenziellen Einsatz von EU-Fördermitteln für makroregionale Strategieprojekte (CPMR 2010).

Makroregionale Strategien liegen im Schnittpunkt unterschiedlicher Systemlogiken. Der Rahmen zwingt die Akteure zu einer Auseinandersetzung über Systemgrenzen hinweg - sowohl horizontal zwischen den Staaten und Politikbereichen als auch vertikal zwischen den unterschiedlichen Ebenen und Institutionen; auch zwischen Politik, Verwaltung, Wirtschaft, Wissenschaft, Zivilgesellschaft und den „Akteuren in der Praxis".

Die bisherigen Erfahrungen und Einschätzungen des Mehrwerts makroregionaler EU-Strategien sind dementsprechend unterschiedlich. Deutlich wird, dass sowohl der Faktor „Zeit“ wie auch diverse makroregions-spezifische Kontexte eine große Rolle spielen. Einheitliche Ansätze für alles können nicht das Ziel sein, die Auswirkungen nicht über einen Leisten geschlagen werden. Ein makroregionaler Strategieprozess ist als Transfer angebotener Möglichkeiten in den Kontext der jeweiligen Makroregion zu begreifen, wobei bisherige Erfahrungen in anderen Makroregionen genützt werden. Die Abwägung von Aufwand und potenziellem Nutzen in jeder einzelnen Region entscheidet letztlich darüber, ob makroregionale EU-Strategien oder andere Optionen gewählt werden. Keineswegs müssen sie flächendeckend auf ganz Europa angewandt werden.

Das hohe $\mathrm{Ma}$ an Offenheit, Pragmatismus und Flexibilität im Ansatz erfordert nichtsdestotrotz eine schrittweise Fundierung der Analyse- und Evaluierungsmethodik. Der Mehrwert makroregionaler EU-Strategien bedarf zunehmend einer schlüssigen Begründung. Es wird notwendig sein, in den Wirkungsmodellen nach unterschiedlichen Logiken differenzierte, aber untereinander kohärente Methoden und Aussagekategorien zu erarbeiten und auch die Brücke zwischen dem Streben der Politik nach schnellen Erfolgen in Form vorweisbar „umgesetzter Projekte“ oder „eingesetzter Förderbeträge“"1) und einem fachlich fundierten Monitoring, das zur Weiterentwicklung des Ansatzes notwendig ist, zu schlagen.

\footnotetext{
1) EU-Kommissar Johannes Hahn sprach anlässlich der 2. Jahreskonferenz zur EU-Donauraumstrategie von ca. 500 Projekten und einem durch die EU-Donauraumstrategie ,bewegten“ Fördervolumen von zirka $€ 49$ Mrd.!
} 


\section{Die bisherigen Methoden einer Analyse und Bewertung der Wirkungen makroregionaler EU-Strategien kämpfen mit komplexen Systemschnittstellen}

Evaluierungen der makroregionalen EU-Strategien sind noch rar, deren methodische Ansätze noch heterogen oder in ihrem eigenen Disziplindenken verhaftet und damit noch bruchstückhaft. Das Methodenverständnis zu makroregionalen EUStrategien befindet sich ebenfalls erst in der Pilotphase. Symptomatisch für diesen Entwicklungsbedarf ist Erhard BusEKs Verteidigung der EU-Donauraumstrategie in der Europäischen Rundschau 4/2013: „Jetzt schon eine makroregionale Strategie als nachvollziehbar zu verlangen und hier Bilanz zu ziehen, ist schlicht und einfach zu früh. [...] Es muss erkannt werden, dass die Initiative davon abhängig ist, in vielen kleinen Teilen vorzugehen, quasi Steine zu einem Mosaik zu fügen, wobei man auf der einen Seite einen Masterplan und auf der anderen Seite die entsprechend bodennahe Aktivität braucht.“ (BusEK 2013) Diese Replik ist natürlich im Kontext der langjährigen politisch-historischen Erfahrungen im Donauraum, gerade auch des Autors selbst, zu verstehen (BUSEK \& GJORESKA 2010), sollte jedoch aus fachlich-methodischer Sicht nicht auf das außen- und integrationspolitisch durchaus legitime Argument „Ein Mehr an Kooperation auf gleicher Augenhöhe ist im Donauraum in jedem Fall ein Erfolg!“ reduziert werden. In einer Region, die erhöhter politischer Aufmerksamkeit bedarf, geht es auch um die Interpretationshoheit. Diese kann zwischen den verschiedenen Systemlogiken unterschiedlich sein. Aus fachlicher Sicht besteht gerade angesichts des gesteigerten politischen Interesses und Gestaltungswillens (wie im Entstehungsprozess der EU-Alpenraumstrategie auch bei Regionalpolitikern zu beobachten) die dringende Notwendigkeit, fundierte fachliche Einschätzungen einer integrierten Gesamtsicht gegenüberzustellen. Angesichts der zeitlich bis dato nicht limitierten Prozesse bei überall begrenzten Ressourcen kann eine von allen Partnern akzeptierte Bewertungsmethode auch die Grundlage zur Einstellung, Fokussierung oder Prioritätensetzung bei einem Prozess sein.

Die EU-Kommission folgte in ihrem Bericht zum Mehrwert makroregionaler Strategien (EuropäIsche Kommission 2013/2), der sich vor allem auf die Strategien im Ostseeraum und im Donauraum bezieht (EuropäIsche Kommission 2013/1), folgenden Evaluierungskriterien: Ergebnisse in Bezug auf konkrete Projekte, Maßnahmen und Initiativen, bessere Politikentwicklung, verbessertes Kosten-Nutzen-Verhältnis, Beseitigung regionaler Ungleichheiten, Förderung der Multi-Level-Governance, verbesserte Zusammenarbeit mit den Nachbarstaaten. Einleitend wird betont, dass „die Auswirkungen der beiden vorhandenen - relativ neuen - makroregionalen Strategien noch schwer zu beurteilen sind und mittel- bis langfristig bewertet werden müssen und zwischen dem Wert des Gesamtkonzepts und Umsetzungsfragen unterschieden werden sollte." Diese interne Evaluierung der EU-Kommission bildete eine Grundlage für die Pressemitteilung des EU-Kommissars vom 9.4.2014 zu den Fortschritten in der EU-Donauraumstrategie, dass „ein äußerst guter Start hingelegt wurde, [...] dass gemeinsam bei der Lösung von Problemen weit mehr erreicht wurde als jedes Land für sich genommen [...] und dass jetzt ein Gang höher geschalten werden muss.“ 
Der erst seit wenigen Jahren geführte fachliche Diskurs zu makroregionalen Strategien führt weitere Analyse- und Bewertungskriterien an, ohne aber bereits eine kohärente, der tatsächlichen Breite des Politikansatzes entsprechende Methodik vorlegen zu können. Makroregionale Strategien werden hierbei in den Kontext der Debatten zu functional geographies versus political realities (DüHR 2011), Territoriality and Soft Planning (FAludi 2013), Soft Spaces, soft planning (Stead 2013, SielKer 2012), rescaling Europe (BIALASIEWICZ et al. 2012), Better Territorial Governance in Europe (NordREgio et al. 2014, BöHME 2013), transformative potentials (DüHR 2011), makroregionale Identitäten (WARNKE 2013), unity versus diversity (BUSEK \& GJORESKA 2010), etc. gestellt.

Die meisten Arbeiten betonen die Multidimensionalität von makroregionalen Strategien samt deren Doppellogik eines politischen (im Sinne von politics) und fachlichen (im Verständnis von policies) Politikansatzes. Es wird auch gefordert, bisherige methodische Modelle, Konzepte und Erfahrungen in einen neuen Kontext zu stellen und nicht den Fehler zu begehen, bisherige Erfahrungen auszublenden (BIALASIEWICZ et al. 2012), sowie diese disziplinenübergreifend anzureichern und zu kombinieren.

Im Auftrag des Bundeskanzleramts wurde im Jahr 2012 ein erster Erfahrungsbericht zur EU-Donauraumstrategie erarbeitet (Метіs GмвH 2012), der folgenden Fragen nachging: Welche österreichischen Akteure sind involviert (Governance)? Was trägt Österreich zur Umsetzung der Strategie bei (Aktivitäten)? Was trägt Österreich zur Finanzierung der Strategie bei (Ressourcen)?

Ausgangspunkt dieser Untersuchung war die These, dass es Zweck einer makroregionalen Strategie sei, einen neuen territorialen Handlungsrahmen zur Lösung bekannter Herausforderungen, als Instrument zur Priorisierung von Handlungsoptionen des öffentlichen Sektors sowie zur Orientierung und Erhöhung der Aufmerksamkeit zu schaffen. Letzteres wurde im Sinne des „Stiftens produktiver Unruhe“ als besonders wertvoll eingestuft. Ergänzend zur allgemeinen Schlussfolgerung, dass unter den beteiligten und für die angesprochenen Themen relevanten Akteuren in der EUDonauraumstrategie ein Mehrwert gesehen wird, sich aber Akteure außerhalb eher abwartend verhalten, postuliert der Bericht, dass eine erfolgreiche Umsetzung der Strategie letztlich nicht so sehr an der Anzahl EU-finanzierter Projekte zu messen sein werde, sondern eher an der Qualität der entwickelten Aktionen (policies), der Reaktion nationaler (und wo vorhanden subnationaler) Regierungen auf die Strategieinhalte und, last but not least, - trotz des Beharrens auf den drei No-s (,keine neuen Strukturen, kein zusätzliches EU-Geld, kein zusätzlicher Rechtsrahmen“ vgl. SAMiECKi 2009) am Umbau der bestehenden transnationalen Organisationen im Sinne institutioneller Innovationen.

Analyse und Bewertung multidisziplinärer Wirkungsdimensionen haben unmittelbare Bedeutung für die Steuerbarkeit und Steuerungsnotwendigkeit makroregionaler EU-Strategien sowie - ganz grundsätzlich - für die Konzeptualisierung des Ansatzes als komplexes System. 


\section{Ausblick: Wieviel Offenheit benötigen und wie viel Verbindlichkeit vertragen makroregionale EU-Strategien?}

Makroregionale Strategien sind durch ein hohes Maß an Spontaneität gekennzeichnet und führen - in der Theorie - zur Herausbildung von vorher nicht konkret planbaren, neuen Systemqualitäten in Folge des Zusammenspiels ihrer Elemente. Makroregionale Strategien liegen fernab eines mechanistischen, deterministischen Politikverständnisses und lassen genügend Raum für spontane Selbstorganisation an einer Vielzahl von Schnittstellen, die Disziplinen, Fachpolitiken und Akteursebenen übergreifen. Neue Verbindungen zwischen den Systemelementen, deren Wirkungsbeziehungen und Prozessverläufen werden zwar angestoßen, sind aber als Einzelelemente kaum von oben planbar. Das Zusammenspiel zwischen Interventionen und Wirkungen auf der Metaebene mit der operativen Mikroebene ist offensichtlich, im Detail jedoch nicht in Form von hierarchisch organisierbaren Ursache-Wirkungs-Beziehungen fassbar. So können makroregionale Strategien längerfristig durchaus unter Beachtung der drei No-s zu neuen Strukturen in der Großregion, zu zusätzlichem finanziellen Mitteleinsatz und zu neuen transnational ausgerichteten Regulativen führen (DüHR 2011).

Das systemische Grundverständnis von makroregionalen EU-Strategien hat unmittelbare Bedeutung für die Ausgestaltung des Steuerungsmodells. Die Strategien sind weder mit der Steuerungslogik eines EU-Förderprogramms, noch jener für internationale Vertragswerke zu erschließen. Sie müssen als neues kooperatives komplexes Wirkungsgefüge verstanden werden. Aber wie viel Offenheit für Anregungen von unten will man zulassen oder kann man aktiv nutzen? Wie viel Steuerung von oben will man übernehmen und kann man mit den vorhandenen Ressourcen und dem politischem Willen tatsächlich leisten? Das sind Fragen, die sich in der Praxis auf jeder Ebene der Mitwirkung stellen - von der Ausgestaltung der strategischen Koordinationsrolle der EU-Kommission bis hin zur Mitwirkungsmöglichkeit zivilgesellschaftlicher Akteure bei den einzelnen Aktionen oder der Involvierung von Parlamenten und Landtagen in die makroregionale Strategiearbeit.2)

Die EU-Kommission hat in ihrem Bericht zu „Governance von makroregionalen Strategien“ vom 20.5.2014 explizite Vorschläge für die Weiterentwicklung der Governance auf der Makro- und Mikroebene gemacht (EuropäIsche Kommission 2013/2). Die Tendenz geht in Richtung einer etwas stärkeren Institutionalisierung und Überprüfbarkeit. Die EU-Kommission drängt nach mehr leadership and ownership durch die beteiligten Staaten. Mit der Ausrichtung der Förderprogramme des Zieles „Territoriale Zusammenarbeit“ (ETZ) in der Periode 2014-2020 auf die Geographie der bestehenden Makroregionen sowie ihrer Forderung, die von makroregionalen Strategien berührten Staaten und Regionen mögen diese in allen relevanten EU-Programmen der Europäischen Struktur- und Investitionsfonds (ESIF) prominent verankern, hat die EU-Kommission ihrerseits Akzente für eine stärkere Umsetzungsorientierung gesetzt.

\footnotetext{
2) So wird zum Beispiel der Schwedische Reichstag regelmäßig über den Fortschritt bei der EU-Ostseestrategie informiert.
} 
Allerdings waren die Staaten und Regionen im Ostsee- und Donauraum bisher kaum bereit diesbezüglich radikalere Reformschritte zu setzen. Die europäische Ebene (vor allem EU-Kommission, aber auch Europäisches Parlament und Ausschuss der Regionen) wird daher in der strategischen Gesamtkoordination weiterhin eine große Rolle spielen. Freiräume und Offenheit der Strategieprozesse werden mittelfristig ebenfalls erhalten bleiben, aber mit neuen Elementen zur Stabilisierung und Konsolidierung des Ansatzes in Balance zu halten sein. In der operativen Arbeit wird ein Mindestmaß an stabilen finanziellen und organisatorischen Rahmenbedingungen für die Kooperation über Jahre hinweg gesichert werden müssen. Grundsätzlich ist zu erwarten, dass weiterhin die je nach Land, Thema, Institution, Akteur und Zeit unterschiedlichen Kontexte maßgeblichen Einfluss auf die Weiterentwicklung der makroregionalen EUStrategien haben werden. Neue makroregionale EU-Strategien wie zum Beispiel die EU-Alpenraumstrategie (GLOERSEN et al. 2013) werden diese Fragen regionsspezifisch neu zu beantworten haben.

In der Praxis variiert das Steuerungs- und Koordinationsverständnis sowohl in den einzelnen Themenbereichen der Strategien als auch in der Herangehensweise an die nationale Koordination. Auch hier spielt der politische und kompetenzrechtliche Kontext im jeweiligen Staat eine nicht zu unterschätzende Rolle.

Im föderalen System Österreichs wirken öffentliche Akteure der Bundes- und Landesebene gleichrangig und in Eigenverantwortung gemäß der verfassungsrechtlichen Kompetenzlage an der Strategie mit. Das Bundeskanzleramt (BKA) fungiert gemeinsam mit dem Außenministerium (BMEIA) als nationaler Koordinator, wobei das BKA primär die fachliche und nationale Koordination wahrnimmt und das BMEIA die makroregionale Strategiearbeit in die österreichische Außenpolitik einbettet, und der Außenminister sowohl innerhalb der Bundessregierung als auch nach außen als politisch Verantwortlicher auftritt.

Das österreichische Koordinationsmodell zu den makroregionalen EU-Strategien zielt auf eine intensive Informationsvernetzung unter den handelnden Akteuren ab (gemäß ÖROK-Partnerschaftsmodell: Bundesministerien, Länder, Gemeinde- und Städtebund, Sozialpartner) und ermöglicht es so vor allem „Multiplikatoren“ sich in ihren Institutionen an der Erarbeitung gemeinsamer Sichtweisen zu beteiligen. In diesem Sinne erfüllt die nationale Koordination eine Informations-, Vernetzungs- und Plattformfunktion ohne formale Beschlusskompetenzen. Es besteht die Tendenz, im Rahmen vorhandener Ressourcen auch ausgewählte relevante Akteure aus dem Nichtregierungsbereich und der Zivilgesellschaft in diesen Koordinationsmechanismus einzubeziehen. Mit der nationalen Koordination makroregionaler EU-Strategien ist in Österreich keinerlei Verschiebung von Kompetenzen verbunden. 


\section{Schlussbetrachtung: Positive Emotionalität als Antwort auf die hohe Komplexität in der transnationalen Zusammenarbeit?}

Offensichtlich ist gerade in der Anfangsphase der Faktor „Mensch“ und dessen Bereitschaft und Befähigung zu engagiertem Handeln von zentraler Bedeutung. Allerdings variiert dieser Faktor innerhalb des Governance-Systems stark und kann nicht als abgesichert angenommen werden (GÄNZLE \& WulF 2014). Positive Herangehensweise, Kreativität, kommunikative und kooperative Fähigkeiten, die Fähigkeit Vertrauen aufbauen zu können, das Anerkennen von kleinen Erfolgen und ähnliche sozial-psychologische Faktoren sind gerade in diesen Zusammenhängen wichtig.

Makroregionale EU-Strategien inspirieren außerdem zu positiven Narrativen, Metaphern und Assoziationen - im Unterschied zu den wegen ihres überbordenden Bürokratismus in ihrem Image beschädigten EU-Programmen. So ist es zum Beispiel nicht verwunderlich, dass das Instrument bisher vor allem in Grenzräumen der EU eingesetzt wird, wobei Nicht-EU-Staaten ,auf Augenhöhe“ kooperieren können. Es kann visionäre Zukunftsbilder anregen, aber wohl auch historisch-kulturelle Reminiszenzen. Wahrscheinlich ist dies auch ein Grund, warum makroregionale Strategien für die Politik attraktiv sind. Außenminister Michael Spindelegger sprach zum Beispiel in seiner Presseaussendung anlässlich der Präsentation des Fortschrittsberichts zur EU-Donauraumstrategie am 10.4.2013 von der „,Donauraumstrategie als Österreichs Verbindung mit der Zukunftsregion Schwarzes Meer“. Die EU-Donauraumstrategie eröffnet ohne Zweifel gerade für Österreich eine weitere Möglichkeit, außen- und integrationspolitische Ziele in Südosteuropa und wirtschaftspolitische Interessen in diesem Raum mit konkreter Zusammenarbeit an transnationalen Entwicklungsthemen zu verbinden (CHRISTEN 2014).

Eine positive Konnotation auf der Metaebene ist jedoch nicht nur für die Politik wichtig, sondern stärkt auch den Akteuren den Rücken und eröffnet ihnen weitere Handlungsmöglichkeiten. Der EU-Kontext sichert hochrangig verankerte Prozessstabilität in der Dreieckskonstellation EU - Staat - Thema und sorgt laufend für Innovationsimpulse.

\section{Literaturverzeichnis}

Bialasiewicz L., Giaccaria P., Jones A., Minca C. (2012), Re-scaling Europe: EU macro-regional fantasies in the Mediterranean. In: European Urban and Regional Studies, 20, 1, S. 59-76.

Böнме K. (2013), Added values of macro regional strategies: a governance perspective (= Spatial Foresight Brief, 3). Luxembourg, Spatial Foresight. - http://www.spatialforesight. $e u$ (abgefragt am 20.06.2014).

Busek E. (2013), Wirkliche Wirklichkeit der EU-Donauraumstrategie. In: Europäische Rundschau, 4, S. 87-89.

Busek E., Guoreska A. (2010), The Danube Region: Transformation and emergence. In: Eastern Journal of European Studies, 1, S. 9-20.

Christen E. (2014), Österreichs außenwirtschaftliche Beziehungen mit den westlichen Balkanländern. In: WIFO-Monatsberichte, 87, 3, S. 197-211. 
Conference of Peripheral Maritime Regions of Europe (CPMR) (Hrsg.) (2010), What opportunities do territorial cooperation and the macro-regional strategies represent for cohesion policy after 2013? Technical paper from the General Secretariat of the Conference of Peripheral Maritime Regions of Europe (CPMR). Rennes.

Dubois A., Hedin S., Schmitt P., Sterlin J. (2009), EU macro-regions and macro-regional strategies - A scoping study (= Nordregio Electronic Working paper, 4). Stockholm, Nordregio.

DüHR S. (2011), Baltic Sea, Danube and Macro-Regional Strategies: A model for Transnational Cooperation in the EU? Brüssel, Notre Europe.

EuropäISCHE KOMmISSION (Hrsg.) (2008), Grünbuch zum Territorialen Zusammenhalt (COM(2008) 616final), Bericht der Kommission an das Europäische Parlament, den Rat, den Europäischen Wirtschafts- und Sozialausschuss und den Ausschuss der Regionen vom 6.10.2008. Brüssel.

EuropäIsche Kommission (Hrsg.) (2013/1), Bericht der Kommission an das Europäische Parlament, den Rat, den Europäischen Wirtschafts- und Sozialausschuss und den Ausschuss der Regionen vom 8.4.2013 zur Strategie der Europäischen Union für den Donauraum (COM(2013)181 final). Brüssel.

Europäische Kommission (Hrsg.) (2013/2), Bericht der Kommission an das Europäische Parlament, den Rat, den Europäischen Wirtschafts- und Sozialausschuss und den Ausschuss der Regionen vom 27.6.2013 zum Mehrwert makroregionaler Strategien (COM(2013) 468final). Brüssel.

EuropäIsche Kommission (Hrsg.) (2014), Bericht der Kommission an das Europäische Parlament, den Rat, den Europäischen Wirtschafts- und Sozialausschuss und den Ausschuss der Regionen vom 20.5.2014 zur Governance makroregionaler Strategien (COM(2014)284final). Brüssel.

FALUdi A. (2013), Territorial cohesion, territorialism, territoriality and soft planning: a critical view. In: Environment and Planning A, 45, 6, S. 1302-1317.

Gänzle S., Wulf J. (2014), The Emerging Core of the EU Macro-regional Governance Architecture (= ISL working paper, 1). Kristiansand, University of Agder, Department of Political Science and Management.

Gloersen E., Bausch Th., Dal Fiore F., Hurel H., Pfefferkorn W., Ratti C., Zavodnik-Lamovsek A. (2013), Strategy-development for the Alpine Space, final report produced by an expert team on behalf of the ETC Alpine Space programme 2007-2013. - http://www. alpine-space.eu (abgefragt am 20.06.2014).

Görmar W. (2010), Makroregionale Strategien: Eine neue Dimension europäischer Zusammenarbeit? In: Informationen zur Raumentwicklung, 8, S. 577-589.

Mетіs GmвH (Hrsg.) (2012), Österreich und die EU-Strategie für den Donauraum: Erfahrungen und Perspektiven im ersten Umsetzungsjahr, Expertinnenbericht im Auftrag des Bundeskanzleramts. - http://www.oerok.gv.at (abgefragt am 20.06.2014).

Nordregio, Delft University of Technology, Politecnico di Torino, University of Newcastle upon Tyne, Centre for Regional Studies of the Hungarian Academy of Sciences, UniVERSity of Ljubljana (Hrsg.) (2014), Towards better Territorial Governance in Europe. A guide for practitioners, policy and decision makers based on contributions from the ESPON TANGO project, ESPON report. - http://www.espon.eu (abgefragt am 20.06.2014).

SAmiecki P. (2009), Macro-regional strategies in the European Union. Discussion paper presented by Commissioner Pawl Samiecki in Stockholm on 18.9.2009.

Sielker F. (2012), Makroregionale Strategie der EU und Soft Spaces - Perspektiven an der Donau. Diplomarbeit, Technische Universität Dortmund.

STEAD D. (2013), European Integration and Spatial Rescaling in the Baltic region: Soft Spaces, Soft Planning and Soft Security (= European Planning Studies). Delft, OTB Research Institute, Delft University of Technology.

W ARNKE K. (2012), Die Strategie der Europäischen Union für den Donauraum - Versuch einer kritischen Bewertung aus der Sicht des Anrainerstaats Bayern. Diplomarbeit, Universität Augsburg. 